\title{
Role of Cnot6l in maternal mRNA turnover
}

\author{
Filip Horvat ${ }^{1,2, \star} \mathbb{D}$, Helena Fulka ${ }^{1,3, \star}$, Radek Jankele ${ }^{1, \star}$, Radek Malik ${ }^{1} \mathbb{D}$, Ma Jun ${ }^{4,5}$, Katerina Solcova', Radislav Sedlacek \\ Kristian Vlahovicek ${ }^{2}$ (D), Richard M Schultz ${ }^{4,5}$, Petr Svoboda ${ }^{1}$ (D)
}

\begin{abstract}
Removal of poly(A) tail is an important mechanism controlling eukaryotic mRNA turnover. The major eukaryotic deadenylase complex CCR4-NOT contains two deadenylase components, CCR4 and CAF1, for which mammalian CCR4 is encoded by Cnot6 or Cnot6l paralogs. We show that Cnot6l apparently supplies the majority of CCR4 in the maternal CCR4-NOT in mouse, hamster, and bovine oocytes. Deletion of Cnot6l yielded viable mice, but $\mathrm{Cnot}^{-1-}$ females exhibited $\sim 40 \%$ smaller litter size. The main onset of the phenotype was post-zygotic: fertilized Cnot6l ${ }^{-l-}$ eggs developed slower and arrested more frequently than $\mathrm{Cnot6l}^{+/-}$eggs, suggesting that maternal CNOT6L is necessary for accurate oocyte-to-embryo transition. Transcriptome analysis revealed major transcriptome changes in $\mathrm{Cnot6l}^{-{ }^{-1-}}$ ovulated eggs and one-cell zygotes. In contrast, minimal transcriptome changes in preovulatory $\mathrm{Cnot}^{-l-} \mathrm{I}^{-1}$ oocytes were consistent with reported Cnot $6 \mathrm{l}$ mRNA dormancy. A minimal overlap between transcripts sensitive to decapping inhibition and Cnot6l loss suggests that decapping and CNOT6L-mediated deadenylation selectively target distinct subsets of mRNAs during oocyte-to-embryo transition in mouse.
\end{abstract}

DOI 10.26508/Isa.201800084 | Received 5 May 2018 | Revised 4 July 2018 | Accepted 5 July 2018 | Published online 16 July 2018

\section{Introduction}

During the oocyte-to-embryo transition (OET), maternal mRNAs deposited in the oocyte are gradually replaced by zygotic mRNAs. Consequently, control of mRNA stability is a principal mechanism assuring correct gene expression reprogramming at the beginning of development. Maternal mRNA degradation during mouse OET occurs in several distinct waves (reviewed in detail in references 1 and 2). Control of mRNA stability involves various mechanisms target, many employing protein interaction with the $3^{\prime}$ untranslated region, that ultimately target the terminal $5^{\prime}$ cap and 3' poly $(A)$ tail structures (reviewed in reference 3 ). The main mammalian mRNA decay pathway involves deadenylation coupled with decapping (4). Eukaryotic cells employ three main deadenylases: CCR4-NOT (carbon catabolite repression 4-negative on TATA-less) complex, PAN2/3 complex, and PARN, which differ in sensitivity to cap structure, poly $(A)$ tail length, and poly(A)binding protein (PABP) (reviewed in references 5 and 6). Cytoplasmic mRNA decay in mammalian cells initiates at the $3^{\prime}$ end and involves sequential deadenylation, first by PAN2/3 followed by CCR4-NOT $(4,7)$. However, recent data suggest that CCR4NOT-mediated deadenylation is the main pathway in general mRNA turnover (8).

The multiprotein CCR4-NOT complex (reviewed in references 9 and 10) was first identified in Saccharomyces cerevisiae as a gene regulating glucose-repressible alcohol dehydrogenase 2. The mammalian CCR4-NOT complex (Fig 1A) is composed of a docking platform (CNOT1) that binds regulatory components (CNOT2, CNOT3, CNOT4, CNOT9, CNOT10, and CNOT11) and two deadenylase components equivalent to yeast's CAF1 and CCR4 deadenylases. CAF and CCR4 differ with respect to their relationship with the PABP; CCR4 can degrade poly $(A)$ bound with PABP, whereas CAF1 degrades free poly(A) (8). Mammals use two paralogs of CAF1 (CNOT7 and CNOT8) and two of CCR4 (CNOT6 and CNOT6L). Thus, a CCR4-NOT complex carries one of four possible combinations of CAF1 and CCR4 homologs. However, the significance of different CCR4-NOT variants remains unclear.

The CCR4-NOT complex can be recruited to mRNA by different BTG/Tob proteins, selective RNA-binding proteins such as tristetraprolin, or upon miRNA binding through TNRC6A-C proteins (reviewed in reference 11). Although miRNA-mediated mRNA degradation is insignificant (12), BTG4 plays a major role in maternal mRNA degradation (13). Another mechanism of selective mRNA targeting by CCR4-NOT is direct recruitment of the complex through YTHDF2, which binds the $N 6$ adenosine $\left(\mathrm{m}^{6} \mathrm{~A}\right)$ RNA modification (14). YTHDF2 was linked to selective elimination of maternal mRNAs during oocyte maturation (15).

Maternal mRNAs in mouse oocytes are unusually stable during the growth phase before oocyte maturation, which is accompanied

${ }^{1}$ Institute of Molecular Genetics of the Czech Academy of Sciences, Prague, Czech Republic ${ }^{2}$ Bioinformatics Group, Division of Molecular Biology, Department of Biology, Faculty of Science, University of Zagreb, Zagreb, Croatia ${ }^{3}$ Institute of Animal Science, Prague, Czech Republic ${ }^{4}$ Department of Biology, University of Pennsylvania, Philadelphia, PA, USA ${ }^{5}$ Department of Anatomy, Physiology, and Cell Biology, School of Veterinary Medicine, University of California, Davis, CA, USA ${ }^{6}$ Czech Centre for Phenogenomics and Laboratory of Transgenic Models of Diseases, Institute of Molecular Genetics of the Czech Academy of Sciences, v. v. i., Vestec, Czech Republic

Correspondence: svobodap@img.cas.cz

Radek Jankele's present address is Swiss Institute for Experimental Cancer Research (ISREC), School of Live Sciences, Swiss Federal Institute of Technology Lausanne (EPFL), Lausanne, Switzerland.

*Filip Horvat, Helena Fulka, and Radek Jankele contributed equally to this work. 


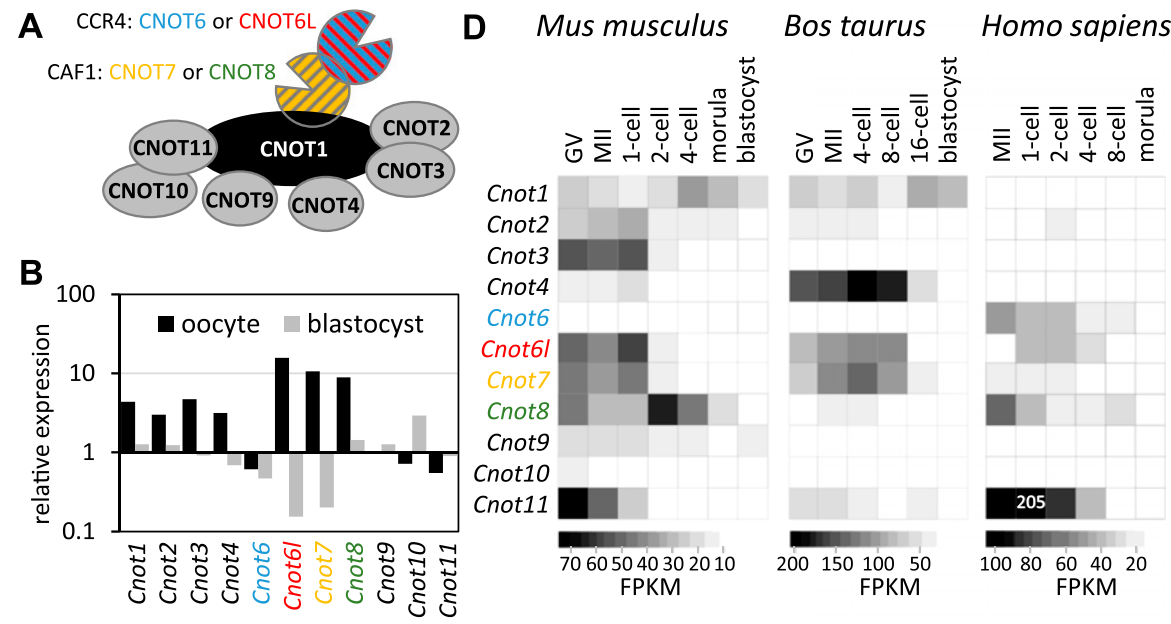

C

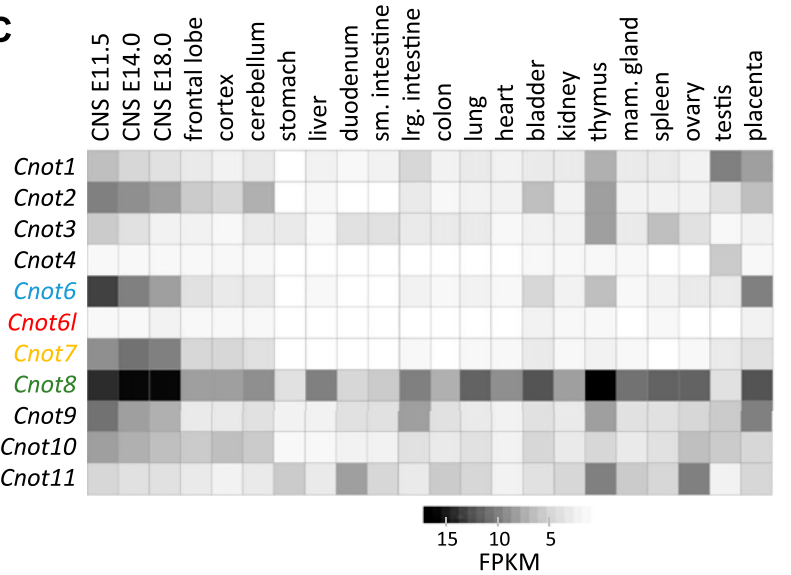

with a transition from mRNA stability to instability (reviewed in reference 2). This transition also involves recruitment of dormant maternal mRNAs that were accumulated but not (or poorly) translated during the growth phase. Some dormant mRNAs encode components of mRNA degradation pathways $(13,16,17)$ and include DCP1A and DCP2, which are critical components of the decapping complex (16). Inhibiting the maturation-associated increase in DCP1A and DCP2 results in stabilizing a subset of maternal mRNAs that are normally degraded and affects zygotic genome activation (16). Dormancy was also shown for BTG4 (13) and components of deadenylase complexes: PAN2 for the PAN2/3 complex and CNOT7 and CNOT6L for the CCR4-NOT complex (17).

Here, we report an analysis of Cnot6l function during OET in mice. Transcripts encoding the CCR4-NOT complex are relatively more abundant in mouse oocytes than in the blastocyst or in somatic tissues. Cnot6l expression apparently supplies the majority of the CCR4 component of the maternal CCR4-NOT complex in mouse, hamster, and bovine, but not human, oocytes. Mice lacking Cnot6l are viable and fertile. However, zygotes arising from $\mathrm{Cnot6l}^{-/-}$eggs develop slower and more likely developmentally arrest than zygotes from heterozygous eggs. Correspondingly, Cnot6l $^{-/-}$females exhibit $\sim 40 \%$ lower fertility. Consistent with the previous report that Cnot6l is a dormant maternal mRNA (17), transcriptome analysis

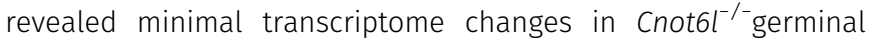

vesicle-intact (GV) oocytes. Nevertheless, there is a subset of maternal mRNAs that are stabilized during oocyte maturation and after fertilization, suggesting that CNOT6L primarily acts in maternal mRNA degradation during oocyte maturation and in zygotes.

\section{Results and Discussion}

\section{Mammalian CCR4 paralog CNOT6L is highly expressed in oocytes}

Several components of the CCR4-NOT complex have a higher relative expression in oocytes in the gcRMA mouse set in the GNF Symatlas database (18) (Fig 1B). Of the four genes encoding active deadenylase components of the CCR4-NOT complex, the CCR4 paralog Cnot6 showed slightly lower expression when compared with a panel of somatic tissues, whereas transcript abundance of the Cnot6l paralog appeared highly enriched in oocytes. These data suggested that CNOT6L could be the main CCR4 deadenylase component during OET (Fig 1B). In contrast, Cnot6 appeared to be highly expressed in many somatic tissues, particularly in embryonic neuronal tissues (Fig 1C).

For further insight into the expression of the CCR4-NOT complex during OET, we analyzed transcript levels of individual components in RNA-sequencing (RNA-seq) data from OET in mouse $(19,20)$, cow 
(21), and human (22) oocytes (Fig 1D). In mouse oocytes, Cnot6l mRNA level was approximately 10 times higher than Cnot6, which is expressed during oocyte growth and is apparently not a dormant maternal mRNA (23). High Cnot6l and low Cnot6 expression during OET was also observed in cow but not in humans, where the level of Cnot6 transcript was higher in metaphase II (MII) eggs. A subsequent equalization of Cnot6 and Cnot6l expression in human zygotes (Fig 1D) could be a consequence of cytoplasmic polyadenylation of dormant Cnot6l mRNA, which can manifest as an apparent increase in mRNA level in poly(A) RNA-seq data (24). High Cnot6l and low Cnot6 expression was also found in GV oocytes of golden hamster, suggesting that this difference in expression is a conserved feature in rodents (Fig 1E). Interestingly, CAF1 paralogs Cnot7 and Cnot8 showed more variable patterns, including equal expression of both paralogs in mouse, dominating Cnot7 in bovine and golden hamster, and dominating Cnot8 paralog expression in human oocytes.

\section{Cnot6l knockout is viable but exhibits reduced fertility}

Given the dominant maternal expression of Cnot6l relative to its paralog Cnot6, we decided to examine the role of Cnot6l in mice using a TAL effector nuclease (TALEN)-mediated knockout. We designed two TALEN pairs, which would induce $\sim 31.3$-kb deletion, affecting exons 5-12 (Fig 2A). This deletion, which would eliminate the entire CC4b deadenylase domain and a part of the upstream leucine-rich repeat region, was expected to genetically eliminate the CNOT6L protein. We obtained two founder animals carrying two very similar deletion alleles (Figs $2 \mathrm{~B}$ and S1). Interestingly, the second allele (Cnot6L-del5-12b) contained a 25-bp insert apparently derived from mitochondrial DNA.
Male and female $\mathrm{Cnot}^{-1 /-}$ mice appeared normal and were fertile. Cnot6l is thus a nonessential gene. Breeding heterozygotes or $\mathrm{Cnot}^{-1 /-}$ males with $\mathrm{Cnot}^{-l^{+/}}$females yielded on average $6.9 \pm 1.6$ and $6.2 \pm 1.9$ pups per litter, respectively (Table 1), which is consistent with the reported $\mathrm{C} 57 \mathrm{BL} / 6$ litter size of $6.2 \pm 0.2$ (25). We typically observe six to eight animals per litter in the C57BL/ 6 strain used to produce mouse models in our facility (20). Analysis of the Cnot6l $\mathrm{I}^{-1-}$ breeding data showed that an average litter size of approximately four pups of $\mathrm{Cnot6l}^{-1-}$ females mated with $\mathrm{Cnot}^{+l^{++}}$, $\mathrm{Cnot}^{+l^{+-}}$, or $\mathrm{Cnot}^{-1-}$ males (Table 1). Reduced litter sizes of $\mathrm{Cnot6l}^{-1-}$ females that mated with males of any of the three genotypes were statistically significant $(P<0.01$, two-tailed $t$ test) when compared with the litter size of $\mathrm{Cnot}^{+/-}$animals. The breeding data thus indicated a maternal-effect phenotype and showed no evidence for a significant role of zygotic and embryonic expression of Cnot6l.

Superovulated knockout females yielded on average the same number of MII eggs as heterozygote littermates and wild-type C57BL/ 6 (31.5 versus 32.3 versus 28.5 , respectively; $n=6$ ), suggesting that the reduction of litter size occurs during fertilization or after fertilization. Accordingly, we analyzed early development of zygotes derived from Cnot6l ${ }^{-1-}$ and Cnot6l $^{+/-}$eggs fertilized in vitro with wild-type sperm and observed a small but significant reduction of fertilization efficiency of $\mathrm{Cnot}^{-1 /-}$ versus $\mathrm{Cnot6l}^{+/-}$eggs (85 versus 99\%; 133/155 Cnot6l $^{-/-}$versus 203/205 Cnot6l $^{+/-}$eggs that formed zygotes; Fisher's test $P$-value $<0.001)$. Analysis of the cleavage times of embryos using a PrimoVision time-lapse system revealed a small but significant delay in early development that could contribute to the reduced litter size (Fig 2C). Importantly, although there was no stage-specific arrest of development for $\mathrm{Cnot} \mathrm{l}^{-1-}$-fertilized eggs, they were two times more likely to fail to reach the blastocyst stage than their $\mathrm{Cnot} \mathrm{I}^{+/-}$-derived counterparts
A

Cnot6I mRNA, variant 1 (NM_144910.2): chr5:96,070,333-96,161,990 (-)

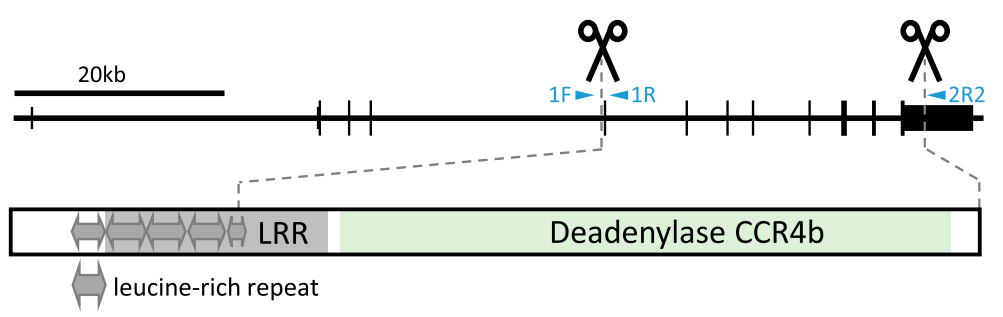

B

Cnot $6 \mathrm{~L}-\mathrm{de} 15-12 \mathrm{a}$

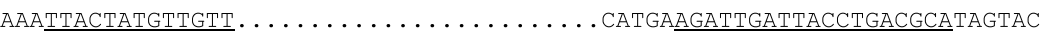

Cnot $6 \mathrm{~L}-\mathrm{de} 15-12 \mathrm{~b}$

AAATTACTATGTTGTTCTATAACATtattaacattattattacaggtatatGATTACCTGACGCATAGTAC

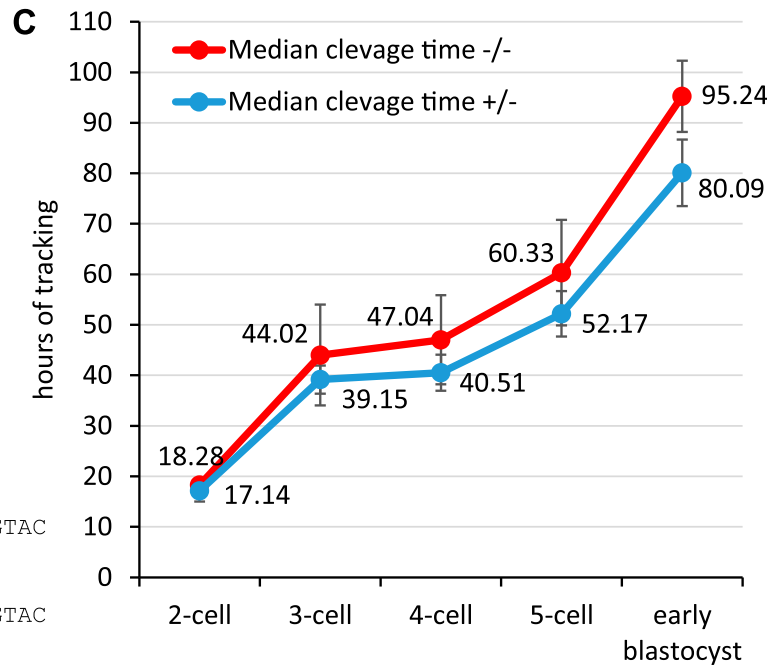

embryonic stage/cleavage event

Figure 2. TALEN-mediated knockout of Cnot6l gene in mice.

(A) A scheme of Cnot6l gene depicting the position of the deletion in the genomic DNA and the corresponding part of the CNOT6L protein. Protein domains were mapped using the Conserved Domain Database search (43). (B) Sequences of two alleles identified in $\mathrm{F}_{0}$ animals, which carry $31.3-\mathrm{kb}$ deletions of exons 4-11. Underlined sequences indicate TALEN cognate sequences. A short fragment of mitochondrial DNA integrated into the deleted locus is visualized in lowercase red font. (C) $\mathrm{Zygotes}$ from in vitro-fertilized $\mathrm{Cnot} \mathrm{l}^{-/-}$eggs develop significantly slower ( $t$ test, $P$-value $<0.001$ for all stages) than zygotes developing from heterozygous eggs. Error bar = SD. In total, 95 and 133 zygotes produced from Cnot $6 l^{-/-}(-/-)$and $\mathrm{Cnot}^{-1 /-}(+/-)$, respectively, were analyzed using the PrimoVision time-lapse system. Numbers indicate hours from the point the zygotes were placed into the tracking system ( $5 \mathrm{~h}$ after mixing sperm with cumulus oocyte complex), which automatically detects the first four cleavage events and formation of early blastocysts. 
Table 1. Breeding performance of Cnot6l mutants.

\begin{tabular}{lllllllll}
$\mathbf{F} \times \mathbf{M}$ & $\mathbf{+}+\mathbf{+}$ & $\mathbf{+}-\mathbf{-}$ & $\boldsymbol{- I}-$ & $\mathbf{n . d}$ & $\mathbf{M}$ & $\mathbf{F}$ & Litters & Litter size $( \pm$ SD $)$ \\
\hline$+/-\times+/-$ & 26 & 30 & 18 & 2 & 39 & 37 & 11 & $6.9( \pm 1.6)$ \\
\hline$+/-\times-/-$ & 0 & 22 & 32 & 2 & 28 & 28 & 9 & $6.2( \pm 1.9)$ \\
\hline$-/-\times+/+$ & 0 & 48 & 0 & 1 & 27 & 22 & 13 & $3.8( \pm 1.6)$ \\
\hline$-/-\times+/-$ & 0 & 4 & 11 & 13 & 9 & 19 & 7 & $4.0( \pm 1.4)$ \\
\hline$-/-\times-/-$ & 0 & 0 & 21 & 0 & 9 & 12 & 5 & $4.2( \pm 1.8)$ \\
\hline
\end{tabular}

(38/95 [40.0\%] Cnot6l $^{-1-}$ versus 26/133 [19.6\%] Cnot6l $^{+/-}$-fertilized eggs failed to develop to the blastocyst). This observation suggested reduced developmental competence of $\mathrm{Cnot}^{-l^{-/}}$zygotes and likely accounted for the reduced litter size (Table 1).

\section{Small but significant transcriptome changes in $\mathrm{Cnot}^{-\mathrm{I}^{-1-}}$ oocytes and zygotes}

To explore the impact of Cnot6l loss on the transcriptome during OET, we performed RNA-seq analysis of GV oocytes, MII eggs, and one-cell zygotes. All replicates showed good reproducibility (Figs S2 and S3). RNA-seq data showed minimal levels of transcripts arising from the deleted Cnot6l allele (Figs 3A and S4). In addition, we did not observe any compensatory change in Cnot6 mRNA expression (Fig 3B).

Transcription from the deleted locus yielded low levels of aberrant transcripts where the splice donor of the third coding exon of Cnot6l was spliced with five different downstream splice acceptor sites in the adjacent intron or downstream of the last Cnot6l exon (Fig S4). In all cases, exons spliced with the third coding exon contained stop codons and, thus, all transcripts generated from the deleted locus would encode the truncated CNOT6L protein composed of the $\mathrm{N}$-terminal leucine repeats. It is unlikely that such isoforms would affect fertility as they would also be present in oocytes of $\mathrm{Cnot6l}^{+/-}$females, which have normal fertility.

Principal component analysis indicated a small magnitude of changes in knockout samples as the samples clustered primarily by developmental stages (Fig 3C). Analysis of differentially expressed transcripts using DESeq2 package (26) with the default $P$-value cutoff 0.1 showed minimal transcriptome changes in GV oocytes (only four
A

Cnot6l

chr5:96,069,079-96,166,535

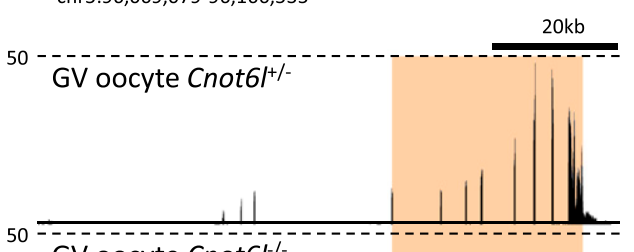

GV oocyte Cnot6/
B

Cnot6

chr11:49,668,768-49,715,504
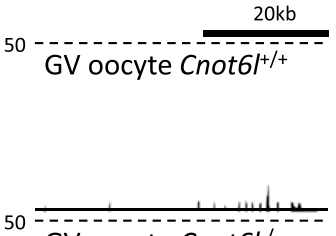

GV oocyte Cnot6 $\%$
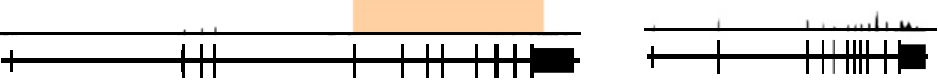

D

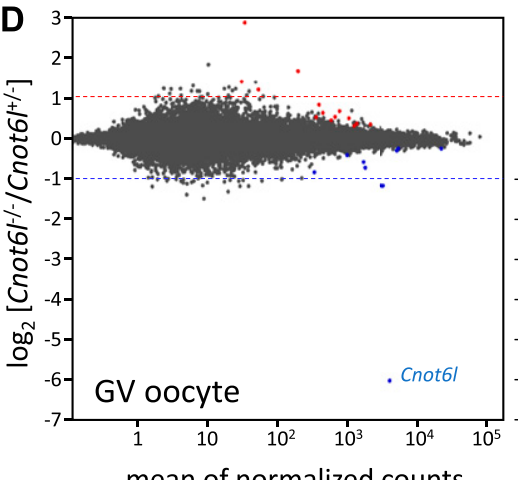

mean of normalized counts

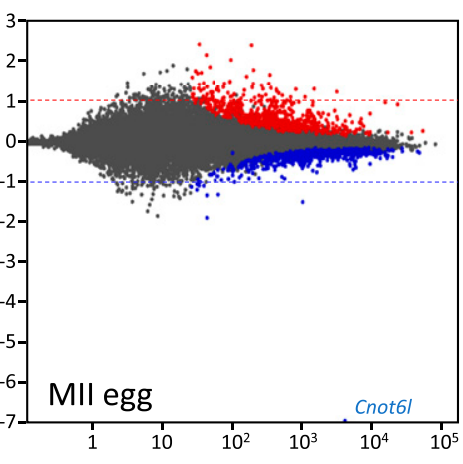

mean of normalized counts

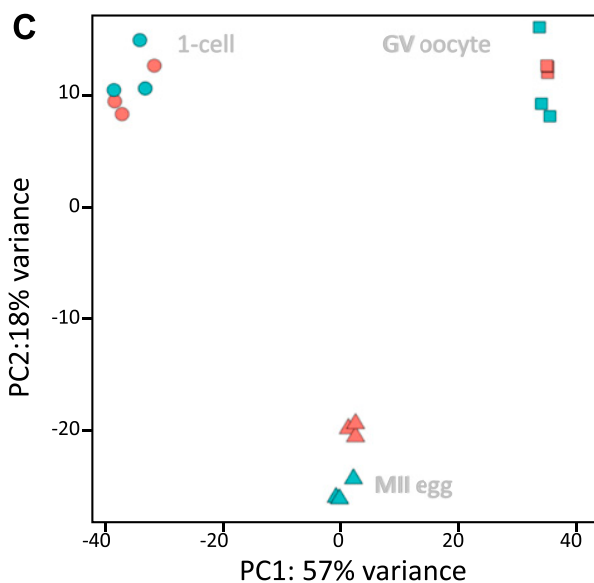

genotype

$>-1-$

$\diamond+/-$

stage

1-cell

$\square$ GV oocyte

$\triangle$ MIl egg

Figure 3. Transcriptome changes in Cnot6l knockout oocytes and zygotes.

(A) Transcriptional landscape in the Cnot6l locus in oocytes from Cnot6l $\mathrm{l}^{+/-}$and Cnot6l $\mathrm{l}^{-/-}$animals. Shown is a UCSC genome browser snapshot (36) of the Cnot6l locus with expression data from one of the replicates of $\mathrm{Cnot}_{6} \mathrm{l}^{+/-}$and $\mathrm{Cnot}_{6 \mathrm{l}^{-/-}}$samples. The orange region indicates the region deleted in knockouts. (B) Loss of $\mathrm{Cnot} 6 \mathrm{l}$ expression has no effect on Cnot6 expression. Shown is a UCSC genome browser snapshot of the Cnot6 locus from the same samples as in panel (A). (C) Principal component analysis of transcriptomes of $\mathrm{Cnot} \mathrm{I}^{-/-}$and control oocytes and zygotes. Heterozygous littermates were used as controls in case of GV and one-cell zygotes; age-matched C57BL/ 6 females were used as controls for MII eggs because there were not enough Cnot6l ${ }^{+/-}$littermates for all control samples. (D) Differentially expressed transcripts in Cnot6l ${ }^{-/-}$GV oocytes, MII $^{-}$ eggs, and one-cell zygotes. MA plots depict genes with significantly higher (red) or lower (blue) mRNA abundance. Dashed lines depict twofold change for easier navigation. The outlier gene at the bottom of each graph is Cnot6l. (E) Venn diagrams depicting numbers of genes showing significantly different transcript abundances in $\mathrm{Cnot6l}^{-/-}$oocytes and zygotes. 
transcripts showing a significant increase in abundance greater than twofold), which indicates that Cnot6l is not required for the formation of the maternal transcriptome. There was, however, an apparent progressive transcriptome disturbance in MII eggs and one-cell zygotes (Fig 3D and Table S1-S3). This finding is consistent with the previously reported dormancy of Cnot6l (17) and the hypothesis that the reduced litter size of $\mathrm{Cnot}^{-1 /-}$ females is a maternal-effect phenotype. The magnitude of transcriptome disturbance appears small despite the number of significantly affected genes; if RNA-seq data would be quantified as transcripts per million, higher transcript levels of 622 genes in MII eggs (Fig 3E) would account for $1.28 \%$ of the transcriptome.

Interestingly, the numbers of significantly up-regulated and down-regulated mRNAs were comparable (Fig 3E), which was unexpected because transcript stabilization would be the primary expected effect of a deadenylase component loss from the CCR4-NOT complex. It is possible that preventing CNOT6L-mediated deadenylation (hence destabilization) of transcripts from several hundred genes might result in accelerated degradation of other transcripts, noting that we previously observed a similar phenomenon when the maturation-associated increase in DCP1A/DCP2 was inhibited (16). In any case, when the significantly stabilized transcripts were projected onto transcriptome changes during maturation and following fertilization, it was clear that exclusive CNOT6Ldependent destabilization of maternal transcripts concerns only a smaller fraction of maternal mRNAs degraded during OET (Fig 4A).

Relative transcript changes during OET can be problematic to interpret because they may reflect changes in poly(A) tail length and not changes in transcript abundance due to mRNA degradation or transcription (24). Although the Ovation system used for producing RNA-seq libraries uses total RNA as input material, genomemapped data show that mRNAs are preferentially sequenced and the sequencing yields a slight bias toward mRNAs with longer poly (A) tails (e.g., Mos mRNA, a typical dormant maternal mRNA polyadenylated during meiotic maturation [27], showed an apparent $\sim 17 \%$ increased abundance in control wild-type MII eggs relative to GV oocytes).

To examine a potential impact of poly $(A)$ tail length on transcript abundance during meiotic maturation, we used a published poly(A) tail sequencing dataset (28) to generate a plot of the relative change in transcript abundance in MII eggs as a function of poly(A) tail length (Fig 4B). These data showed that transcripts showing relatively increased abundance in Cnot6l $^{-1-}$ eggs typically have longer poly $(A)$ tails (60-80 nt). However, when taking into account the distribution of poly $(A)$ lengths in the entire transcriptome, the relative frequency of transcripts with increased abundance in Cnot6l $^{-1-}$ eggs was similar for transcripts with poly(A) tails 30-80 nt in length (Fig 4C), suggesting that Fig 4B data only reflect that most maternal transcripts have poly(A) tails 60-80 nt long.

To further resolve the issue of mRNA abundance versus poly(A)length effects in differentially expressed transcripts in zygotes derived from $\mathrm{Cnot6l}^{-/-}$eggs, we used RNA-seq datasets from MII eggs and one-cell zygotes that were generated from directly selected poly $(A)$ and from total RNA without any poly(A) bias $(19,20)$. These data allow distinguishing between true mRNA degradation, which would be observed in the total RNA data, and deadenylation/ polyadenylation, which would manifest in the poly(A) data (Fig 4D). When transcripts showing a significant relative increase in $\mathrm{Cnot}^{-1} \mathrm{I}^{-}$ zygotes were projected on these data, there was a clear shift to the left on the $x$-axis, consistent with their deadenylation. Furthermore, a fraction of these transcripts also showed apparent degradation as evidenced by their position on the $y$-axis (Fig 4D). Altogether, these data show that maternal Cnot6l contributes to maternal mRNA deadenylation and degradation during OET.
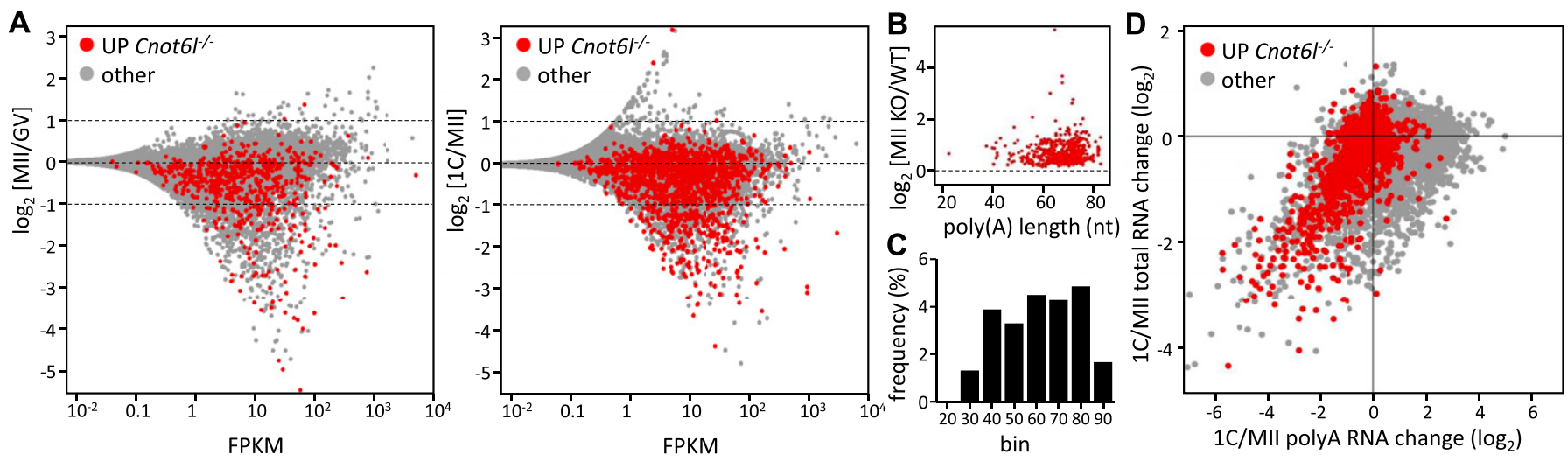

Figure 4. Transcriptome changes in Cnot6l knockout oocytes and zygotes.

(A) Projection of differentially expressed transcripts in Cnot6l ${ }^{-1-}$ MII eggs and zygotes onto transcriptome changes during meiotic maturation and after fertilization. MA

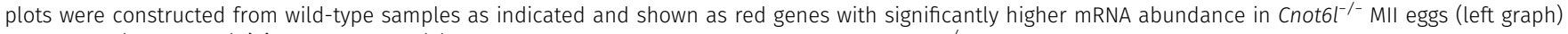
and zygotes (right graph). (B) Analysis of poly(A) tail length of mRNAs significantly increased in Cnot6l ${ }^{-/-}$oocytes during meiosis. The $y$-axis shows the relative up-regulation of maternal mRNAs in Cnot6l $\mathrm{I}^{-/-}$MII eggs (same genes as those labeled in red in MIl eggs in Fig 3D). The $x$-axis depicts poly(A) tail length in GV oocytes taken from the literature (28). (C) Relative distribution of mRNAs significantly increased Cnot6l ${ }^{-1-}$ oocytes according to poly(A) tail length. RNAs were binned according to the poly(A) tail length into 10-nt bins (0-10, 10-20, 20-30, etc.), and the number of transcripts significantly increased in Cnot6l ${ }^{-/-}$MII eggs was divided with the total number of transcripts in each bin. The $x$-axis numbers represent the upper values of binned poly(A) tail lengths. According to the poly(A) length in GV oocytes, the $y$-axis shows the relative up-regulation of maternal mRNAs in Cnot6l ${ }^{-/-}$MII eggs (corresponding to genes labeled in red in MII eggs in Fig 3D). The $x$-axis depicts the poly(A) tail length in GV oocytes taken from the literature (28). (D) Transcriptome changes in $\mathrm{Cnot6l}^{-/-}$zygotes are consistent with a role for CNOT6L in deadenylation during OET. Published RNA-seq data for relative poly(A) RNA and total RNA changes $(19,20)$ were used to construct the plot. In red are shown genes with significantly higher mRNA abundance in Cnot6l ${ }^{-/}$zygotes. The $y^{-}$-axis shows relative changes in total RNA (i.e., RNA degradation), whereas the $x$-axis shows poly(A) RNA changes (i.e., RNA degradation and/or deadenylation). 
Finding that the transcriptome changes following Cnot6l loss are restricted to a fraction of deadenylated and degraded maternal mRNAs suggests some selectivity of a CNOT6L-containing CCR4NOT complex in targeting mRNAs. CCR4-NOT complex recruitment to maternal mRNAs through BTG4 does not appear very selective given the large number of affected maternal mRNAs in $\mathrm{Btg}^{4^{-/-}} \mathrm{MII}$ eggs, which includes $\sim 1 / 3$ of the transcripts showing a relative increase in $\mathrm{Cnot6l}^{-/-}$MII eggs (Fig 5A). These data also suggest that the CAF1 (CNOT7 and CNOT8) component of the CCR4-NOT complex is probably sufficient for a large part of deadenylation mediated by the CCR4-NOT complex. This suggestion is also consistent with the effect of CNOT7 knockdown in early embryos (17), which is apparently more detrimental for early development than the loss of CNOT6L reported here.

To gain further insight into the potential selectivity of CNOT6Lmediated mRNA deadenylation and degradation, we examined overlaps with transcriptome changes in Ythdf2 knockout eggs (15) (Fig 5B), in Tut4/ $/ 7^{-1-}$ oocytes (28) (Fig 5C) and in eggs with suppressed decapping (16) (Fig 5D). In all three cases, transcriptome changes concerned hundreds of transcripts. Accordingly, we assessed whether CNOT6L contributes to selective targeting of $\mathrm{m}^{6} \mathrm{~A}$ marked maternal mRNAs during meiotic maturation and to what extent mRNAs destabilized through CNOT6L and the decapping complex are mutually exclusive. In all cases, the overlap of transcripts whose relative abundance is increased in MII eggs was minimal (although statistically significant in the case of the Ythdf2 knockout [Fisher's exact test $P$-value $=4.06 \mathrm{e}-14]$ ). Furthermore, transcripts regulated by Ythdf2 and Tut4/7 were apparently less expressed during meiotic maturation ( $<10$ FPKM; Fig 5B and C) than transcripts targeted by decapping (Fig 5D).

In any case, maternal mRNAs preferentially targeted through decapping are, thus, a distinct group from those stabilized upon elimination of Cnot6l. This difference becomes apparent when these transcripts are visualized in transcriptome data from unfertilized and fertilized eggs resolved according to relative abundance in total RNA and poly(A) RNA-seq $(19,20)$ (Fig 6). In this display, the $y$-axis corresponds to RNA degradation and the $x$-axis reflects poly $(A)$ changes. Deadenylated and degraded RNAs are found in the lower left quadrant. When transcripts up-regulated in Cnot6l $^{-/-}$MIl eggs or up-regulated in MII eggs upon inhibition of decapping are highlighted in this plot, transcripts most sensitive to decapping inhibition seem to be degraded without pronounced deadenylation, unlike transcripts sensitive to Cnot6l loss (Fig 6).

A selective function has been proposed for CNOT6-mediated deadenylation of maternal mRNAs. CNOT6 is present in full-grown GV oocytes in cortical foci and regulates deadenylation of mRNAs such as Orc6 or Slbp that were transiently polyadenylated during early meiotic maturation (23). Remarkably, CNOT6 and CNOT6L paralogs are highly similar at the protein level (Fig S5); the major differences concern the five-amino-acid residue longer $\mathrm{N}$ terminus of CNOT6L and the five-amino-acid residue insertion in CNOT6 at the end of the $\mathrm{N}$-terminal leucine-rich repeat region, which stems from using an alternative splice donor. Further research should reveal whether these differences underlie any distinct recruitment of CCR4-NOT complexes carrying these paralogs or whether apparent selectivity is determined by other factors, such as the length of the poly(A) tail, differential expression of the paralogs, or their specific localization in oocytes and zygotes.

Taken together, we show that loss of Cnot6l in mice results in reduced fertility. Although we cannot rule out that some of the effects observed in the oocyte or early embryos could be indirect effects of a role for Cnot6l (e.g., in granulosa cells), the phenotype is presumably a consequence of perturbed deadenylation and degradation of maternal mRNAs during OET. Because Btg $4^{-/-}$eggs

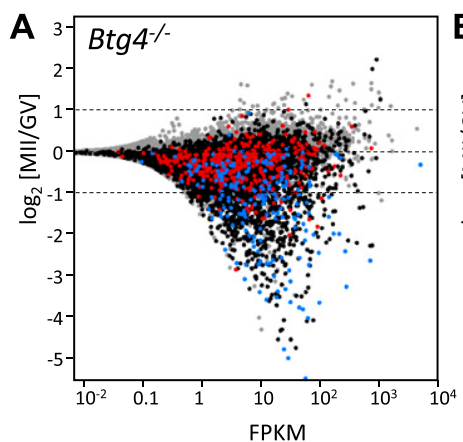

- UP in Cnot61- MII

- UP in Btg $4 \%$ MII

- UP Cnot61\% \& Btg $4^{-/ \mathrm{MII}}$

other

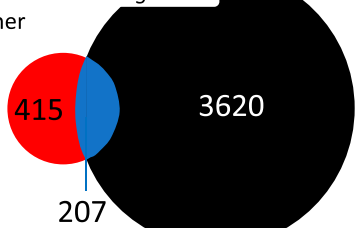

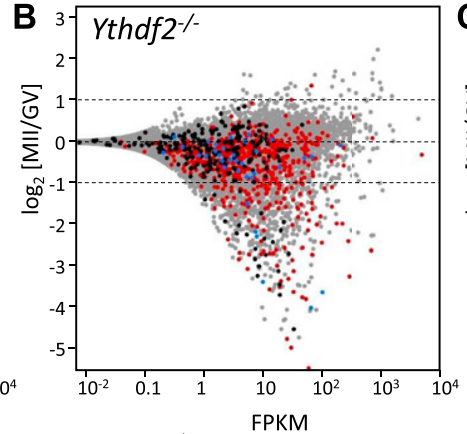

- UP in Cnot6l- MII

- UP $Y$ thdf $2 \%$ MII

- UP Cnot61-- \& Ythdf2-/- MII

other

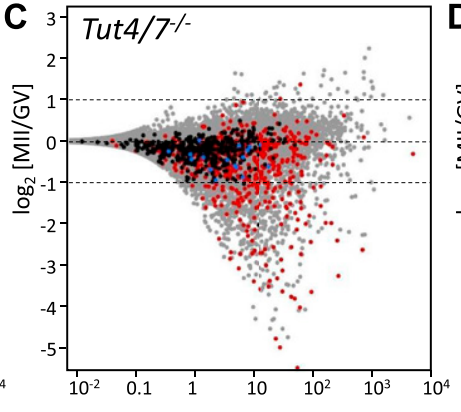

- UP in Cnot61\%- MII

- UP in Tut $4 / 7 \%$ GV

- UP Cnot6/- MII \& Tut4/7/- GV

other

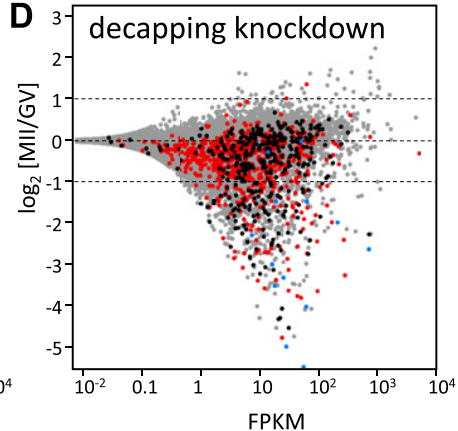

UP in Cnot61\%- MII

- UP decapping knock-down MII

- UP Cnot6/\% \& decapping knock-down MI other

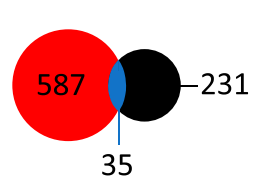

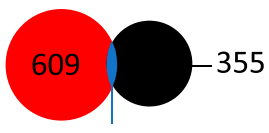

13

Figure 5. Comparison of transcriptome changes in Cnot6l ${ }^{-/-}$MII eggs with other experimental data.

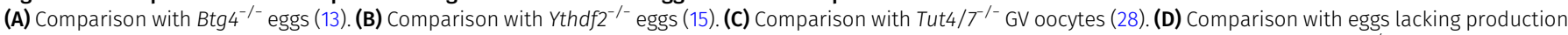
of the decapping complex (16). In each case, significantly up-regulated transcripts in MII eggs were compared with up-regulated transcripts in Cnot6l/- MII eggs (Fig 3D). All MA plots were constructed from wild-type control replicates from GSE116771. 


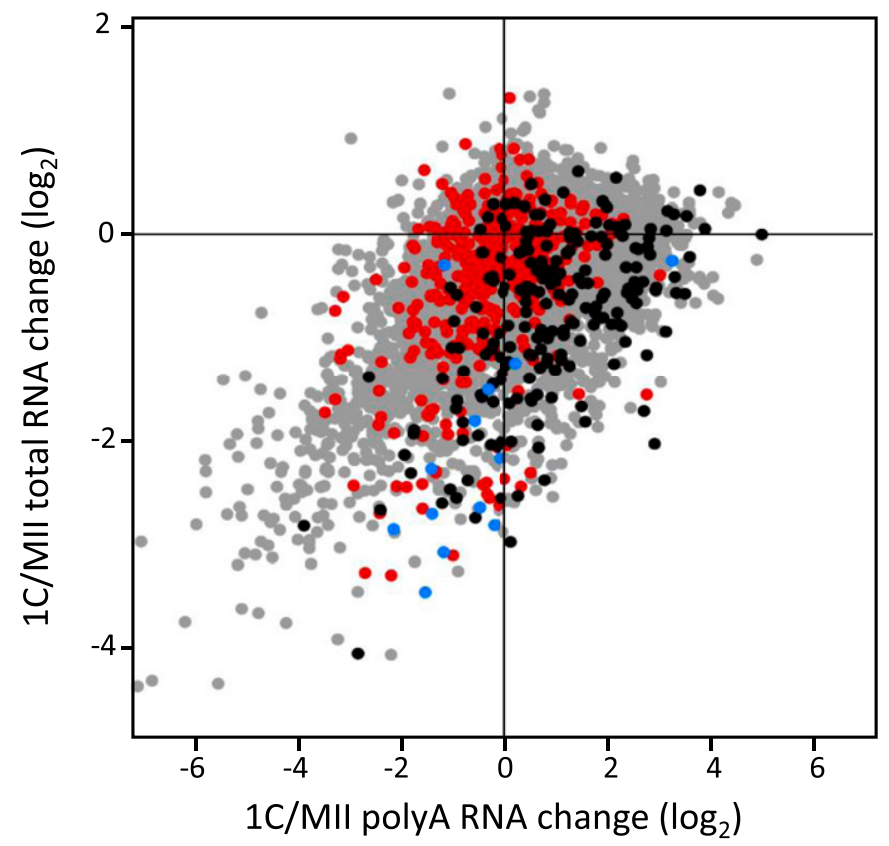

\section{- UP in Cnot6/ $\%$ MII \\ - UP decapping knock-down MII - UP Cnot61\%- \& decapping knock-down MII other}

Figure 6. Comparison of transcriptome changes in $\mathrm{Cnot6l}^{-/-} \mathrm{MII}^{-}$eggs with other experimental data.

Projection of transcripts with relatively increased abundance in Cnot6l ${ }^{-/-}$MII eggs and eggs with blocked decapping (Fig 5D) onto transcriptome changes in zygotes. The plot was constructed from published data $(19,20)$ as in Fig 4D. The $y$-axis shows relative changes in total RNA-seq (i.e., RNA degradation), whereas the $x$-axis shows poly(A) RNA changes from poly(A) RNA-seq (i.e., RNA degradation and/or deadenylation). In red are shown transcripts with significantly higher mRNA abundance in Cnot6l $^{-/-}$MII eggs. In black are shown transcripts with significantly higher mRNA abundance in MII eggs upon inhibition of decapping. In blue are shown transcripts up-regulated in both conditions.

exhibit much larger transcriptome changes than Cnot6l $^{-/-}$eggs, CNOT6L-mediated deadenylation appears rather selective. It is presently unclear if this selectivity stems from truly selective targeting (e.g., dependent on recruitment of the CNOT6L-containing CCR4-NOT complex directly through CNOT6L). Given a possible redundancy with the CAF1 component of the CNOT6L complex and/or other RNA degrading mechanisms, we speculate that a spectrum of transcripts targeted by the CNOT6L-containing CCR4-NOT complex is much broader and that transcripts showing a relative increase upon loss of Cnot6l are less targeted by redundant mechanisms.

\section{Materials and Methods}

\section{Oocyte and embryo collection}

Oocytes and early embryos were obtained from superovulated mice as described previously (29). Resumption of meiosis during culture of GV oocytes was prevented with $0.2 \mathrm{mM}$ 3-isobutyl-1-methylxanthine (Sigma-Aldrich). Animal experiments were approved by the Institutional Animal Use and Care Committees (approval no. 024/2012) and were carried out in accordance with the European Union regulations.

\section{Production of Cnot6l knockout model}

Cnot6l knockout mice were produced by the Transgenic and Archiving Module of the Czech Centre for Phenogenomics (http:/ www. phenogenomics.cz/), Institute of Molecular Genetics ASCR, using TALENs (reviewed in reference 30) designed to delete coding exons 4-11 (Fig 2A). TALEN plasmids were produced as described previously (31). Target sites for TALEN pairs inducing an $\sim 31.3-\mathrm{kb}$ deletion (chr5: $96,075,067-96,106,332$ [GRCm38/mm10]) were identified using the SAPTA tool (32) and TALEN off-targeting was addressed with the PROGNOS tool (33). The following RVD repeats were used to generate individual TALENs:

C6L-1R repeats NH NI HD NI NI NG NG NH HD NI NI NG NG NG HD(cognate sequence: TO GACAATTGCAATTTC)

C6L-1F repeats NG NI HD NG NI NG NH NG NG NH NG NG HD NG(cognate sequence: TO TACTATGTTGTTCT)

C6L-4F repeats NG NI NG NI NG NI HD HD NG HD NI NI NH HD NI NG HD HD-(cognate sequence: TO TATATACCTCAAGCATCC)

C6L-4R repeats NH HD NH NG HD NI NH NH NG NI NI NG HD NI NI NG HD NG-(cognate sequence: TO GCGTCAGGTAATCAATCT)

TALEN RNAs for injection were produced as described previously (31). A sample for microinjection was prepared by mixing all four TALEN RNAs in ultrapure water at a concentration of $4 \mathrm{ng} / \mu \mathrm{l}$ each. This mixture was loaded into the injection capillary and injected into male pronuclei of C57BL/ 6 one-cell embryos.

Genotyping was performed by PCR on lysates from tail biopsies from 4-wk-old animals using genotyping primers Cnot6l-1F (5'-GTCATCAGGTTTGGCAGCAAGC-3') and Cnot6l -1R (5'-CTAAGAAGTGTGTGGTGCATCAGC-3') for the wild-type allele (yielding a 597-bp product) and Cnot6l-1F and Cnot6l-2R2 (5'-CAGAGAAGAAAGCCCACCCG-3') for the deletion (yielding a predicted 357-bp product).

\section{Analysis of preimplantation development}

Mice were superovulated and cumulus oocyte complexes were isolated as described previously (29). Sperm of C57BL/6) (8-12 wk old) males were used for in vitro fertilization. Males were euthanized by cervical dislocation and sperm were isolated from cauda epididymis, capacitated for $1 \mathrm{~h}$ in human tubal fluid medium, and mixed with cumulus oocyte complexes in human tubal fluid. In vitro fertilization was performed for $5 \mathrm{~h}$. Next, zygotes containing two pronuclei were selected and preimplantation development was analyzed by the PrimoVision time-lapse system (Vitrolife) with 15-min acquisition settings ( $\mathrm{t}=0$; start of recording). Embryos were cultured in $\mathrm{KSOM}$ medium at $37^{\circ} \mathrm{C}$ under $5 \% \mathrm{CO}_{2}$ until the blastocyst stage. Automatically recorded times for preset cleavage events were confirmed by personal inspection and the median time was plotted against the embryonic stage. The experiment was repeated five times. Heterozygous and homozygous females used in each 
experiment were littermates, the eggs were fertilized by a single male, and embryos were developed side-by-side in a single incubator. Representative recorded videos (Videos 1 and 2) are provided in the Supplementary Information.

\section{RNA-seq}

Total RNA was extracted from triplicates of 25 wild-type or knockout GV oocytes, MII eggs, or one-cell zygotes using a PicoPure RNA isolation kit with on-column genomic DNA digestion according to the manufacturer's instructions (Thermo Fisher Scientific). Each sample was spiked in with $0.2 \mathrm{pg}$ of synthesized Renilla luciferase mRNA before extraction as a normalization control. Non-stranded RNA-seq libraries were constructed using the Ovation RNA-seq system V2 (NuGEN) followed by the Ovation Ultralow Library system (DR Multiplex System; NuGEN). RNA-seq libraries were pooled and sequenced by 125 -bp paired-end reading using the Illumina HiSeq at the High Throughput Genomics Core Facility at the University of Utah, Salt Lake City, UT. RNA-seq data were deposited in the Gene Expression Omnibus database under accession ID GSE116771.

\section{Bioinformatics analyses}

\section{Mapping of Illumina RNA-seq reads on the mouse genome}

All RNA-seq data were mapped using the STAR mapper (34) version 2.5.3a as described previously (35), except all multi-mapping reads:

STAR -readFilesIn \$FILE1 \$FILE2 -genomeDir \$GENOME_INDEX -runThreadN 8 -genomeLoad LoadAndRemove -limitBAMsortRAM 20000000000 -readFilesCommand unpigz -c -outFileNamePrefix \$FILENAME -outSAMtype BAM SortedByCoordinate-outReadsUnmapped Fastx -outFilterMultimapNmax 99999 -outFilterMismatchNoverLmax 0.2 -sjdbscore 2.

The following genome versions were used for mapping the data: mouse-mm10/GRCm38, human-hg38/GRCh38, cow-bosTau8/UMD3.1, and hamster-MesAur1.0 (GCF_000349665.1).

Annotated gene models for all organisms corresponding to their respective genome versions were downloaded from the Ensembl database as gene transfer format files. Only protein-coding genes were used in all subsequent analyses. Data were visualized in the University of California, Santa Cruz (UCSC), genome browser by constructing bigWig tracks using the UCSC tools (36).

\section{Differential expression analysis of RNA-seq data}

Analysis of genes differentially expressed in knockouts compared with wild types in different developmental stages was performed in the $\mathrm{R}$ software environment. Mapped reads were counted over exons grouped by gene as follows.

GenomicAlignments::summarizeOverlaps(features = exons, reads = bamfiles, mode $=$ "Union", singleEnd $=$ FALSE, ignore.strand $=$ TRUE).

Statistical significance and fold changes in gene expression were computed using the DESeq2 package (26) from RNA-seq data prepared as biological triplicates. Briefly, DESeq2 analysis starts with a matrix of read counts obtained with summarizeOverlaps() command above, in which each row represents one gene and each column one sample. Read counts are first scaled by a normalization factor to account for differences in sequencing depth between samples. Next, dispersion (i.e., the variability between replicates) is calculated for each gene. Finally, negative binomial generalized linear model (GLM) is fitted for each gene using those estimates and normalized counts. GLM fit returns coefficients, indicating the overall expression strength of the gene and coefficients (i.e., $\log _{2}{ }^{-}$ fold change) between treatment and control (in our analysis knockout and wild-type samples). Significance of coefficients in GLMs are tested with the Wald test. Obtained $P$-values are adjusted for multiple testing using the Benjamini and Hochberg False Discovery Rate procedure (37). In our analysis, expression changes with p-adjusted values smaller than 0.1 (the default DESeq2 cutoff) were considered significant.

\section{Differential expression analysis of microarray data (decapping complex, YTHDF2)}

Microarray data were normalized and background-corrected using RMA (38) (Ythdf2 data) or GC-RMA (39) (decapping complex data) algorithms. Statistical significance and fold changes in gene expression were computed using SAM method (40).

\section{Principal component analysis plot}

Principal component analysis was computed on count data transformed using regularized logarithm (rlog) function from the DESeq2 (26) R package.

\section{Statistical analysis}

Fisher's exact test was used to evaluate the significance of number of genes showing increased transcript abundance in $\mathrm{Cnot6l}^{-/-} \mathrm{MII}$ eggs and MII eggs with reduced decapping complex or knockouts of Ythdf2 or Btg4. A level of $P<0.05$ was considered to be significant.

\section{Supplementary Information}

Supplementary Information is available at https://doi.org/10.26508/lsa. 201800084

\section{Acknowledgements}

We thank Vedran Franke and Josef Pasulka for help with data analysis. This research was supported by the Czech Science Foundation (CSF) grant P305/ 12 /G034 and by the Ministry of Education, Youth, and Sports (MEYS) project NPU1 L01419. Additional support of coauthors included CSF grant 17-08605S to H Fulka; LM2015040 (Czech Centre for Phenogenomics), CZ.1.05/1.1.00/ 02.0109 (Biotechnology and Biomedicine Centre of the Academy of Sciences and Charles University), and CZ.1.05/2.1.00/19.0395 (higher quality and capacity for transgenic models) support by MEYS and RVO 68378050 by the Academy of Sciences of the Czech Republic to R Sedlacek; the European Structural and Investment Funds grant for the Croatian National Centre of Research Excellence in Personalized Healthcare (contract KK.01.1.1.01.0010), Croatian National Centre of Research Excellence for Data Science and Advanced Cooperative Systems (contract KK.01.1.1.01.0009), and Croatian Science Foundation (grant IP-2014-09-6400) to K Vlahovicek; and a grant from National Institutes of Health (HD022681) to RM Schultz.

\section{Author Contributions}

F Horvat: conceptualization, data curation, software, formal analysis, supervision, funding acquisition, investigation, visualization, 
project administration, and writing-original draft, review, and editing

H Fulka: resources, formal analysis, investigation, and writing-review and editing.

R Jankele: resources, data curation, software, formal analysis, investigation, and writing-review and editing.

R Malik: conceptualization, funding acquisition, investigation, project administration, and writing-review and editing.

J Ma: data curation, software, formal analysis, investigation, and writing-review and editing.

K Solcova: investigation, methodology, and writing-review and editing.

R Sedlacek: resources, formal analysis, investigation, and writing-review and editing.

K Vlahovicek: resources, data curation, and software.

RM Schultz: conceptualization, funding acquisition, methodology, project administration, and writing-review and editing.

P Svoboda: conceptualization, formal analysis, supervision, funding acquisition, investigation, visualization, project administration, and writing-original draft, review, and editing.

\section{Conflict of Interest Statement}

The authors declare that they have no conflict of interest.

\section{References}

1. Despic V, Neugebauer KM (2018) RNA tales: How embryos read and discard messages from mom. J Cell Sci 131: jcs201996. doi:10.1242/ jcs.201996

2. Svoboda P, Franke V, Schultz RM (2015) Sculpting the transcriptome during the oocyte-to-embryo transition in mouse. Curr Top Dev Biol 113: 305-349. doi:10.1016/bs.ctdb.2015.06.004

3. Houseley J, Tollervey D (2009) The many pathways of RNA degradation. Cell 136: 763-776. doi:10.1016/j.cell.2009.01.019

4. Yamashita A, Chang TC, Yamashita Y, Zhu W, Zhong Z, Chen CY, Shyu AB (2005) Concerted action of poly(A) nucleases and decapping enzyme in mammalian mRNA turnover. Nat Struct Mol Biol 12: 1054-1063. doi:10.1038/nsmb1016

5. Parker R, Song H (2004) The enzymes and control of eukaryotic mRNA turnover. Nat Struct Mol Biol 11: 121-127. doi:10.1038/nsmb724

6. Goldstrohm AC, Wickens M (2008) Multifunctional deadenylase complexes diversify mRNA control. Nat Rev Mol Cell Biol 9: 337-344. doi:10.1038/nrm2370

7. Bartlam M, Yamamoto T (2010) The structural basis for deadenylation by the CCR4-NOT complex. Protein Cell 1: 443-452. doi:10.1007/s13238-0100060-8

8. Yi H, Park J, Ha M, Lim J, Chang H, Kim VN (2018) PABP cooperates with the CCR4-NOT complex to promote mRNA deadenylation and block precocious decay. Mol Cell 70: 1081-1088.e1085. doi:10.1016/j. molcel.2018.05.009

9. Collart MA, Panasenko OO (2017) The Ccr4-Not complex: Architecture and structural insights. Subcell Biochem 83: 349-379. doi:10.1007/978-3319-46503-6_13

10. Collart MA (2016) The Ccr4-Not complex is a key regulator of eukaryotic gene expression. Wiley Interdiscip Rev RNA 7: 438-454. doi:10.1002/ wrna.1332
11. Doidge R, Mittal S, Aslam A, Winkler GS (2012) Deadenylation of cytoplasmic mRNA by the mammalian Ccr4-Not complex. Biochem Soc Trans 40: 896-901. doi:10.1042/bst20120074

12. Suh N, Baehner L, Moltzahn F, Melton C, Shenoy A, Chen J, Blelloch R (2010) MicroRNA function is globally suppressed in mouse oocytes and early embryos. Curr Biol 20: 271-277. doi:10.1016/j.cub.2009.12.044

13. Yu C, Ji SY, Sha QQ, Dang Y, Zhou JJ, Zhang YL, Liu Y, Wang ZW, Hu B, Sun QY, et al (2016) BTG4 is a meiotic cell cycle-coupled maternal-zygotictransition licensing factor in oocytes. Nat Struct Mol Biol 23: 387-394. doi:10.1038/nsmb.3204

14. Du H, Zhao Y, He J, Zhang Y, Xi H, Liu M, Ma J, Wu L (2016) YTHDF2 destabilizes $\mathrm{m}(6) \mathrm{A}$-containing RNA through direct recruitment of the CCR4-NOT deadenylase complex. Nat Commun 7: 12626. doi:10.1038/ ncomms12626

15. Ivanova I, Much C, Di Giacomo M, Azzi C, Morgan M, Moreira PN, Monahan J, Carrieri C, Enright AJ, O'Carroll D (2017) The RNA m(6)A reader YTHDF2 is essential for the post-transcriptional regulation of the maternal transcriptome and oocyte competence. Mol Cell 67: 1059-1067.e1054. doi:10.1016/j.molcel.2017.08.003

16. Ma J, Flemr M, Strnad H, Svoboda P, Schultz RM (2013) Maternally recruited DCP1A and DCP2 contribute to messenger RNA degradation during oocyte maturation and genome activation in mouse. Biol Reprod 88: 11. doi:10.1095/biolreprod.112.105312

17. Ma J, Fukuda Y, Schultz RM (2015) Mobilization of dormant Cnot7 mRNA promotes deadenylation of maternal transcripts during mouse oocyte maturation. Biol Reprod 93: 48. doi:10.1095/biolreprod.115.130344

18. Su Al, Cooke MP, Ching KA, Hakak Y, Walker JR, Wiltshire T, Orth AP, Vega RG, Sapinoso LM, Moqrich A, et al (2002) Large-scale analysis of the human and mouse transcriptomes. Proc Natl Acad Sci USA 99: 4465-4470. doi:10.1073/pnas.012025199

19. Abe K, Yamamoto R, Franke V, Cao M, Suzuki Y, Vlahovicek K, Svoboda P, Schultz RM, Aoki F (2015) The first murine zygotic transcription is promiscuous and uncoupled from splicing and 3' processing. EMBO J 34: 1523-1537. doi:10.15252/embj.201490648

20. Karlic R, Ganesh S, Franke V, Svobodova E, Urbanova J, Suzuki Y, Aoki F, Vlahovicek K, Svoboda P (2017) Long non-coding RNA exchange during the oocyte-to-embryo transition in mice. DNA Res 24: 129-141. doi:10.1093/dnares/dsx008

21. Graf A, Krebs S, Zakhartchenko V, Schwalb B, Blum H, Wolf E (2014) Fine mapping of genome activation in bovine embryos by RNA sequencing. Proc Natl Acad Sci USA 111: 4139-4144. doi:10.1073/pnas.1321569111

22. Xue Z, Huang K, Cai C, Cai L, Jiang CY, Feng Y, Liu Z, Zeng Q, Cheng L, Sun YE, et al (2013) Genetic programs in human and mouse early embryos revealed by single-cell RNA sequencing. Nature 500: 593-597. doi:10.1038/nature12364

23. Vieux KF, Clarke HJ (2018) CNOT6 regulates a novel pattern of mRNA deadenylation during oocyte meiotic maturation. Sci Rep 8: 6812. doi:10.1038/s41598-018-25187-0

24. Svoboda P, Fulka H, Malik R (2017) Clearance of parental products. Adv Exp Med Biol 953: 489-535. doi:10.1007/978-3-319-46095-6_10

25. Nagasawa H, Miyamoto M, Fujimoto M (1973) Reproductivity in inbred strains of mice and project for their efficient production (author's transl) (in Japanese). Jikken Dobutsu 22: 119-126. doi:10.1538/ expanim1957.22.2_119

26. Love MI, Huber W, Anders S (2014) Moderated estimation of fold change and dispersion for RNA-seq data with DESeq2. Genome Biol 15: 550. doi:10.1186/s13059-014-0550-8

27. Gebauer F, Xu W, Cooper GM, Richter JD (1994) Translational control by cytoplasmic polyadenylation of c-mos mRNA is necessary for oocyte maturation in the mouse. EMBO / 13: 5712-5720. doi:10.1002/j.1460-2075.1994.tb06909.x

28. Morgan M, Much C, DiGiacomo M, Azzi C, Ivanova I, Vitsios DM, Pistolic J, Collier P, Moreira PN, Benes V, et al (2017) mRNA 3' uridylation and poly(A) 
tail length sculpt the mammalian maternal transcriptome. Nature 548: 347-351. doi:10.1038/nature23318

29. Nagy A (2003) Manipulating the Mouse Embryo: A Laboratory Manual. Cold Spring Harbor, NY: Cold Spring Harbor Laboratory Press.

30. Jankele R, Svoboda P (2014) TAL effectors: Tools for DNA targeting. Brief Funct Genomics 13: 409-419. doi:10.1093/bfgp/elu013

31. Flemr M, Malik R, Franke V, Nejepinska J, Sedlacek R, Vlahovicek K, Svoboda P (2013) A retrotransposon-driven dicer isoform directs endogenous small interfering RNA production in mouse oocytes. Cell 155: 807-816. doi:10.1016/j.cell.2013.10.001

32. Lin Y, Fine EJ, Zheng Z, Antico CJ, Voit RA, Porteus MH, Cradick TJ, Bao G (2014) SAPTA: A new design tool for improving TALE nuclease activity. Nucleic Acids Res 42: e47. doi:10.1093/nar/gkt1363

33. Fine EJ, Cradick TJ, Zhao CL, Lin Y, Bao G (2014) An online bioinformatics tool predicts zinc finger and TALE nuclease off-target cleavage. Nucleic Acids Res 42: e42. doi:10.1093/nar/gkt1326

34. Dobin A, Davis CA, Schlesinger F, Drenkow J, Zaleski C, Jha S, Batut P, Chaisson M, Gingeras TR (2013) STAR: Ultrafast universal RNA-seq aligner. Bioinformatics 29: 15-21. doi:10.1093/bioinformatics/bts635

35. Franke V, Ganesh S, Karlic R, Malik R, Pasulka J, Horvat F, Kuzman M, Fulka H, Cernohorska M, Urbanova J, et al (2017) Long terminal repeats power evolution of genes and gene expression programs in mammalian oocytes and zygotes. Genome Res 27: 1384-1394. doi:10.1101/ gr.216150.116

36. Kent WJ, Zweig AS, Barber G, Hinrichs AS, Karolchik D (2010) BigWig and BigBed: Enabling browsing of large distributed datasets. Bioinformatics 26: 2204-2207. doi:10.1093/bioinformatics/btq351
37. Benjamini Y, Hochberg Y (1995) Controlling the false discovery rate: A practical and powerful approach to multiple testing. J R Stat Soc Series $B$ Stat Methodol 57: 289-300. doi:10.2307/2346101

38. Irizarry RA, Hobbs B, Collin F, Beazer-Barclay YD, Antonellis KJ, Scherf U, Speed TP (2003) Exploration, normalization, and summaries of high density oligonucleotide array probe level data. Biostatistics 4: 249-264. doi:10.1093/biostatistics/4.2.249

39. Wu ZJ, Irizarry RA, Gentleman R, Martinez-Murillo F, Spencer F (2004) A model-based background adjustment for oligonucleotide expression arrays. J Am Stat Assoc 99: 909-917. doi:10.1198/016214504000000683

40. Tusher VG, Tibshirani R, Chu G (2001) Significance analysis of microarrays applied to the ionizing radiation response. Proc Natl Acad Sci USA 98: 5116-5121. doi:10.1073/pnas.091062498

41. Winkler GS, Balacco DL (2013) Heterogeneity and complexity within the nuclease module of the Ccr4-Not complex. Front Genet 4: 296. doi:10.3389/fgene.2013.00296

42. Yue F, Cheng Y, Breschi A, Vierstra J, Wu W, Ryba T, Sandstrom R, Ma Z, Davis C, Pope BD, et al (2014) A comparative encyclopedia of DNA elements in the mouse genome. Nature 515: 355-364. doi:10.1038/ nature13992

43. Marchler-Bauer A, Derbyshire MK, Gonzales NR, Lu S, Chitsaz F, Geer LY, Geer RC, He J, Gwadz M, Hurwitz DI, et al (2015) CDD: NCBI's conserved domain database. Nucleic Acids Res 43: D222-D226. doi:10.1093/nar/gku1221

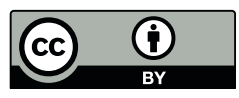

License: This article is available under a Creative Commons License (Attribution 4.0 International, as described at https://creativecommons.org/ licenses/by/4.0/). 